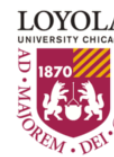

\title{
Body Composition, Serum Biomarkers of Inflammation and Quality of Life in Clinically Stable Women with Estrogen Receptor Positive Metastatic Breast Cancer
}

\author{
Patricia M. Sheean \\ Loyola University Chicago, psheean1@luc.edu \\ Sandra Gomez-Perez \\ Rush University Medical Center \\ Cara Joyce \\ Loyola University Chicago, cjoyce6@luc.edu \\ Vasilios Vasilopoulos \\ Loyola University Medical Center \\ Mary Beth Bartolotta

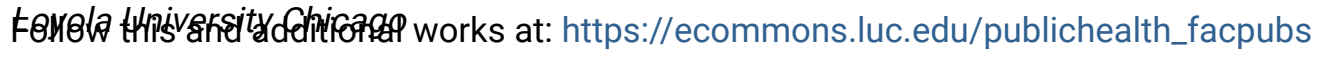 \\ Part of the Public Health Commons
}

See next page for additional authors

\section{Recommended Citation}

Sheean, Patricia M.; Gomez-Perez, Sandra; Joyce, Cara; Vasilopoulos, Vasilios; Bartolotta, Mary Beth; Robinson, Patricia; Lo, Shelly; and Lomasney, Laurie, "Body Composition, Serum Biomarkers of Inflammation and Quality of Life in Clinically Stable Women with Estrogen Receptor Positive Metastatic Breast Cancer" (2019). Parkinson School of Health Sciences and Public Health. 1.

https://ecommons.luc.edu/publichealth_facpubs/1

This Article is brought to you for free and open access by the Faculty Publications and Other Works by Department at Loyola eCommons. It has been accepted for inclusion in Parkinson School of Health Sciences and Public Health by an authorized administrator of Loyola eCommons. For more information, please contact ecommons@luc.edu. \section{(c) $($ ) $\ominus$}

This work is licensed under a Creative Commons Attribution-Noncommercial-No Derivative Works 3.0 License. (c) Taylor and Francis, 2019. 


\section{Authors}

Patricia M. Sheean, Sandra Gomez-Perez, Cara Joyce, Vasilios Vasilopoulos, Mary Beth Bartolotta, Patricia Robinson, Shelly Lo, and Laurie Lomasney 
Title: Body composition, serum biomarkers of inflammation and quality of life in clinically stable women with estrogen receptor positive (ER+) metastatic breast cancer (BC)

\author{
Authors: \\ *Patricia Sheean, PhD, RD; psheean1@luc.edu \\ Sandra Gomez-Perez, PhD, RD ${ }^{\text {b }}$ Sandra_L_gomez-perez@rush.edu \\ Cara Joyce, PhDa; cjoyce6@luc.edu \\ Vasilios Vasilopoulos, AA, AAS, R.T.(R) ${ }^{\text {; }}$; vvasilopoulos@lumc.edu \\ Mary Beth Bartolotta, BSN, RNª; mbartolotta@luc.edu \\ Patricia Robinson, MDc; probinson@lumc.edu \\ Shelly Lo, MD'; slo@lumc.edu \\ Laurie Lomasneyc; Ilomasn@lumc.edu
}

Author affiliations: a Loyola University Chicago; ${ }^{b}$ Rush University Medical Center; ${ }^{\mathrm{c}}$ Loyola University Medical Center

*Corresponding author: Patricia Sheean, PhD, RD, Loyola University Chicago, Marcella Niehoff School of Nursing, 2160 South First Avenue, Building 125, Room 4527, Maywood, IL 60153;

Office 708-216-0344; psheean1@luc.edu

\title{
Statement of Authorship
}

P. Sheean and P. Robinson contributed to the conception and design of the research; P. Sheean, 
M.B. Bartolotta, S. Gomez-Perez, C. Joyce, V. Vasilopoulos, S. Lo, L. Lomasney and P. Robinson contributed to the acquisition and analysis of the data; P. Sheean and C. Joyce contributed to the analyses and interpretation of the data; P. Sheean drafted the manuscript. All authors critically revised the manuscript, agree to be fully accountable for ensuring the integrity and accuracy of the work, and read and approved the final manuscript.

Disclosures: The authors declare that they have no competing interests.

Keywords: sarcopenia, metastatic breast cancer, myosteatosis, body composition, quality of life 
Title: Body composition, serum biomarkers of inflammation and quality of life in clinically stable women with estrogen receptor positive $(E R+)$ metastatic breast cancer $(B C)$

\author{
Abstract: \\ Limited data exist regarding body composition and associated patient-reported outcomes for \\ women with metastatic BC. Demographic, clinical, blood and questionnaire data were collected \\ to quantify body composition and explore associations with symptoms, inflammation and \\ quality of life (QOL) in 41 women with ER+ metastatic BC. Diagnostic/surveillance computed \\ tomography (CT) images including the third lumbar region (L3) were obtained to evaluate \\ skeletal muscle (SM) quantity and quality, and abdominal adipose tissue. Frequencies, medians \\ and interquartile ranges (IQR) are presented, stratified by sarcopenia and obesity (Body mass \\ index $\left.(B M I) \geq 30.0 \mathrm{~kg} / \mathrm{m}^{2}\right)$. Overall, 34\% $(n=14 / 41), 49 \%(n=20 / 41)$ and $34 \%(n=14)$ of women \\ had sarcopenia, myosteatosis and obesity, respectively. Handgrip strength was compromised in \\ $24 \%$ of participants $(n=10 / 41)$. Women with sarcopenia had significantly lower body weight \\ $(p=0.01), B M I(p=<0.001)$, and whole body SM $(p<0.001)$, yet reported greater engagement in \\ leisure time exercises $(p=0.05)$ vs. non-sarcopenic women. Women with obesity had \\ significantly higher levels of abdominal obesity (all values $p<0.0001$ ) and serum biomarkers of \\ inflammation ( $p$ values $<0.06$ ), yet lower QOL $(p=0.02)$ vs. women without obesity. The \\ abPGSGA did not differentiate women with sarcopenia. Future interventions should test if \\ improvements in body composition are associated with better outcomes for this vulnerable, \\ emerging population.
}




\section{Introduction}

Metastatic breast cancer $(\mathrm{BC})$ occurs when malignant cancer cells spread beyond the breast to regional (i.e., lymph nodes) or distant (i.e., bone, liver, brain) areas of the body. (1) While $6 \%$ of women will initially present with metastatic BC, or de novo disease (2), approximately $30 \%$ of women with early stage (I-III) BC will eventually experience disease progression. Metastatic $B C$ is presently incurable, but treatable by means of serial administration of systemic therapies. The goal of treatment in the metastatic setting is to simultaneously maximize disease response, minimize symptom burden and maintain the highest quality of life (QOL). (1) Recent data from the American Cancer Society and the Surveillance, Epidemiology, and End Results (SEER) Program report that only $22-26 \%$ of women initially diagnosed with metastatic BC survive 5 years, with most succumbing to their disease within 2-3 years of metastatic diagnosis. $(2,3)$ However, it is unclear if these data reflect the survival benefits observed from targeted therapies, including cyclin dependent kinase (CDK) 4 and 6 inhibitors or human epidermal growth factor receptor-2 (HER-2). (4, 5) Statistical models from Mariotto et al (6) show that the number of women living with metastatic BC has increased 2-3 fold over the last two decades, especially among those who are younger and have de novo disease. Thus, women with metastatic $B C$ are a growing sector of the cancer survivorship population and we have limited information regarding issues unique to their survivorship.

Approximately $40-60 \%$ of patients present with overweight or obesity at the time of their cancer diagnosis. (7) Unlike other cancer therapies, BC treatment, itself, is associated with weight gain, often resulting in increased levels of adipose tissue with or without the loss of lean mass (LM). (8) This phenomenon is well-documented in women undergoing treatment for early stage 
BC (I-III), (9-14) but not well investigated in women with late stage disease. Due to the routine use of computed tomography (CT) imaging in the metastatic setting, obtaining precise estimates of body composition in this population is achievable. $(15,16)$ However, prior studies have only examined body composition at initial metastatic BC diagnosis and were restricted to outcomes available in the electronic medical record $(\operatorname{EMR}) .(15,16)$ Previously, the lifestyle habits of 25 women with metastatic $\mathrm{BC}$ receiving a variety of cancer therapies at different stages of their disease trajectory were investigated. (17) Forty-four percent $(n=11 / 25)$ voiced concerns regarding unfavorable treatment-related weight gain, as well as infrequent physical activity and dietary intake patterns high in fat and low in fiber. Only surrogate measures of body composition were reported. Building upon this, for the current study we used a convenience sample of women with clinically stable estrogen receptor positive $(E R+)$ metastatic $B C$ to examine the prevalence of compromised SM, specifically sarcopenia and myosteatosis, as well as abdominal adiposity, lifestyle factors, quality of life (QOL) and serum biomarkers of inflammation. We hypothesized that women who reported higher levels of fatigue or lower QOL would exhibit compromises in SM.

\section{Materials and Methods}

This cross-sectional study recruited eligible women from a university-affiliated cancer center (February, 2015 - October, 2017) as part of a Phase 1 vitamin D supplementation trial (NCT02186015). Female participants were required to be: 1$) \geq 18$ years of age, 2 ) undergoing treatment for histologically confirmed estrogen receptor positive (ER+) metastatic $B C, 3)$ at a performance status of 0-2(18) and 4) deemed clinically stable by their medical oncologist (i.e., no unexplained weight loss, no changes in anti-neoplastic agents within the last 30 days, no 
new clinical or radiologic evidence of disease progression, uncontrolled pain or $<6$ months of life anticipated). Informed consent was obtained at a routine clinic visit and anthropometric and questionnaire data were obtained at a separate time. Additional eligibility for the present study included: 1) completion of the study questionnaires, and 2) a chest, abdominal, pelvis CT study including the $\mathrm{L} 3$ region completed as part of the standard of care. This study was approved the Institutional Review Board of Loyola University Chicago.

Demographic and medical information: Demographic (age, race/ethnicity, living situation employment status) and medical information (breast cancer surgery, previous and current cancer treatments, metastatic sites) were gathered from the electronic medical record (EMR) and/or the participant, as appropriate.

Nutrition assessment: During a research visit, height was measured to the nearest $0.1 \mathrm{~cm}$ using a clinic stadiometer (Digital Physician Scale Health O Meter 500KL; McCook, IL) and weight was measured to the nearest $0.1 \mathrm{~kg}$ using a portable digital scale (Tanita BF-350; Arlington Heights, IL). Participants wore light clothes and were measured without shoes. If two measurements were more than $0.5 \mathrm{~cm}$ or $0.2 \mathrm{~kg}$ apart for height and weight, respectively, a third measurement was taken. The two closest measures of height and weight were used to calculate $\mathrm{BMI}\left(\mathrm{kg} / \mathrm{m}^{2}\right.$ ) and to classify obesity status (defined as $\mathrm{BMI} \geq 30.0 \mathrm{~kg} / \mathrm{m}^{2}$ ). (19) Participants completed the abridged Patient Generated Subjective Global Assessment (aPG-SGA); a validated 4 component nutrition assessment tool that assigns numeric values related to weight patterns, food intake, symptoms, and activities and function. Unlike the global Subjective Global Assessment (SGA)(20), the aPG-SGA is self-administered and does not include a physical examination. Higher scores are indicative of greater symptom burden and scores $\geq 6$ points are 
associated with malnutrition. (21) To crudely assess dietary intake, participants completed a validated on-line fruit, vegetable and fiber screener.(22) This 10 item dietary screener takes $\sim 5$ minutes to complete and ranks individuals with regard to their usual intake of fruits and vegetables, providing point estimates of total fruit/vegetable servings and dietary fiber. (23) Output from dietary screener was recorded in hard-copy format. All nutrition assessments were completed prior to CT imaging retrieval and analyses.

Body composition: Generally, imaging studies are completed for surveillance purposes every 36 months for women with ER+ metastatic breast cancer. Imaging is performed more often in women with increased symptoms and suspected disease activity, and less often in women with decreased symptoms and disease stability. The CT image performed for clinical purposes and closest to the participant's baseline study visit was utilized for these analyses. Archived images of the third lumbar (L3) region were identified and anonymized by the Radiology staff. An investigator certified in body composition assessment and blind to outcomes assessment completed the analyses using Slice-O-Matic software (v.4.3; Tomovision, Montreal, Quebec, Canada).

SM quantity: Estimates of whole-body skeletal muscle $(\mathrm{kg})$ were calculated from the regression equation of Mourtzakis et al (24) as follows: $0.30 \times$ [skeletal muscle area at L3 region using CT $\left.\left(\mathrm{cm}^{2}\right)\right]+6.06(r=0.94 ; p<0.001)$. These values were then normalized for stature, height in meters squared $\left(\mathrm{kg} / \mathrm{m}^{2}\right)$. To derive skeletal muscle index (SMI), L3 muscle area measures were normalized by height in meters squared $\left[(\mathrm{SMI}) \mathrm{cm}^{2} / \mathrm{m}^{2}\right]$. To assess the prevalence of CTdetected sarcopenia, we applied the lumbar SMI cut-point for females of $\leq 41.0$ proposed by Martin et al established in larger oncologic patient population.(7) Sarcopenic obesity for 
females was defined as a lumbar $\mathrm{SMI} \leq 41.0 \mathrm{~cm}^{2} / \mathrm{m}^{2}$ and a $\mathrm{BMI} \geq 30 \mathrm{~kg} / \mathrm{m}^{2}$. (The term sarcopenia is used henceforth synonymously with CT-detected sarcopenia.)

SM quality: Skeletal muscle ranges between -29 to +150 Hounsfield units (HU; the CT unit of measure of radiodensity). However, in individuals who are obese (25) or have type 2 diabetes mellitus (26), SM attenuation values are lower symbolizing fatty infiltration of the muscle and a reduced oxidative capacity. (27) Myosteatosis was defined as $<41 \mathrm{HU}$ for $\mathrm{BMI}<24.9 \mathrm{~kg} / \mathrm{m}^{2}$ and $<33 \mathrm{HU}$ for $\mathrm{BMI} \geq 25.0 \mathrm{~kg} / \mathrm{m}^{2}$, using the cut-points of Martin et al. (7)

Adipose tissue: Abdominal obesity, specifically visceral adipose tissue (VAT) and subcutaneous adipose tissue (SAT), are associated with elevated serum biomarkers of inflammation in healthy populations.(28) As part of the CT body composition analyses, measurements of VAT and SAT were quantified. Adipose tissues range according to Hounsfield Unit (HU) thresholds, where -150 to -50 are used for VAT and -190 to -30 are used for SAT. Total adipose tissue (TAT) was calculated as sum of the areas of SAT + VAT.

Quality of life (QOL): Women completed the Functional Assessment of Cancer Therapy-Breast (FACT-B) (29) and the Functional Assessment of Cancer Therapy-Endocrine Symptoms (FACTES). (30) These tools measure multidimensional QOL, including four sub-scale domains (physical well-being, social/family well-being, emotional well-being, and functional well-being), in addition to concerns regarding $\mathrm{BC}$ and endocrine symptoms. The FACT-B includes ten additional questions specific to $\mathrm{BC}$, while the FACT-ES contains 18 additional items related to endocrine symptoms; the latter being highly relevant for women with ER+ disease. Maximum scores for these instruments are 144 and 220, respectively, with higher composite scores indicating better QOL. 
Pain: The Brief Pain Inventory-Short Form (BPI) was specifically designed to assess pain in cancer patients. It includes four items measuring the severity of pain in the last 24 hours, plus seven additional items measuring the extent to which pain interferes with life activities. Higher overall scores indicate greater pain experiences. (31)

Fatigue: The Piper Fatigue Scale (PFS) is a validated tool, composed of 22 numerically scaled "0" to "10" items. It measures four dimensions of subjective fatigue, including: behavioral/severity ( 6 items); affective meaning (5 items); sensory ( 5 items); and cognitive/mood (6 items.) Higher overall scores are indicative of greater fatigue. (32)

Physical functioning: Women completed the Godin Exercise Leisure-time Questionnaire; a 4item tool that demonstrates reliability coefficients of 0.8 with $\mathrm{V}_{2}$ measures (33) and allows for the quick quantification of time spent engaged in strenuous, moderate or light activities over a typical 7-day period. In addition, handgrip strength $(\mathrm{kg})$ was measured with a Jamar Plus handgrip dynamometer following standard study procedures. (34) Measures were obtained in duplicate for each participant's non-dominant and dominant hands during their study appointment. Scores of the dominant hand were averaged and the cut-point of $<20 \mathrm{~kg}$ was used to define compromised functioning. (35)

Serum biomarkers of inflammation: Blood biospecimens were acquired prior to routine clinic appointments, processed immediately and stored at $-80^{\circ} \mathrm{C}$ until the time of analyses. ELISA assay kits were purchased from R\&D Systems (Minneapolis, MN) to profile concentrations of tumor necrosis factor- $\alpha$ (TNF- $\alpha$ ) and interleukin-6 (IL-6) and from Aniara Diagnostica (Westchester, $\mathrm{OH}$ ) to quantify C-reactive protein (CRP). All samples were run in duplicate with controls. 
Statistical Analyses: Descriptive statistics were conducted to examine differences in baseline demographics, clinical characteristics, symptom reports and QOL stratified by sarcopenia and/or obesity status using BMI ( $\left.\geq 30.0 \mathrm{~kg} / \mathrm{m}^{2}\right)$. Sensitivity analyses were completed to assess the prevalence of sarcopenia for variable windows of time (e.g., 30, 60 and >60 days) between questionnaire completion and CT imaging. Patient characteristics are presented as medians, interquartile ranges, or counts and percentages. Statistical differences between groups were assessed with Wilcoxon rank-sum tests or Fisher's exact test for continuous and nominal variables, respectively. Analyses were performed with SAS version 9.4 (Cary, NC) and based on the exploratory nature of this study, a $p$ value of $<0.10$ was used to denote trends and statistical significance.

\section{Results:}

A total of 43 women were enrolled in the parent trial (NCT02186015). Of these, 1 woman withdrew completely and 1 participant did not have any available CT images, leaving 41 participants with evaluable data for the present analyses. The average participant was 59.6 ( \pm $11.9)$ years of age and $26.3( \pm 33.8)$ months since her metastatic BC diagnosis. The majority of women were non-Hispanic white (73\%), had metastases to the bone, liver and/or lung, and were employed full or part-time (44\%). As seen in Table 1, women who were classified with CTdetected sarcopenia were significantly older $(p=0.03)$, disproportionately non-Hispanic white $(p=0.02)$ and receiving less targeted therapies compared to women who were non-sarcopenic $(p=0.02)$.

Our sensitivity analyses demonstrated that the prevalence of sarcopenia was $22.7 \%$ $(n=5 / 22), 25.0 \%(n=2 / 8)$ and $63.6 \%(n=7 / 11)$ for women who completed questionnaires within 
30,60 or $>60$ days (61-159 days) of CT imaging, respectively. To address the potential differences for women imaged less frequently, we analyzed our patient reported outcomes of interest for those restricted to 60 days between questionnaire completion and CT imaging. Similar to the larger group $(n=41)$, there were no significant differences between nonsarcopenic vs. sarcopenic women with regard to abPGSGA total scores (4.2 vs. 2.1, $p=0.16$ ), FACT-Breast total score (100 vs. 112, $p=0.21$ ), FACT-Endocrine Symptoms total score (157 vs. 181. $P=0.07$ ), total fatigue ( 4.5 vs. $2.4, p=0.10$ ) and daily pain ( 3.7 vs. $2.6, p=0.29)$.

Table 2 depicts the differences in body composition, lifestyle factors, QOL, symptoms and serum biomarkers of inflammation stratified by sarcopenia. Overall, $34 \%(n=14 / 41)$ of participants were sarcopenic and $49 \%$ ( $n=20 / 41)$ of all women exhibited myosteatosis; $22 \%$ $(n=9)$ exhibited both of these conditions. Women with sarcopenia had significantly lower body weight $(p=0.01)$, BMI $(p=<0.001)$, whole body SM $(p<0.001)$ and abdominal adipose tissue $(p$ values $<0.003$ ), yet they reported significantly greater engagement in leisure time exercise activity $(p=0.05)$, when compared to those without sarcopenia. Dominant handgrip strength was compromised in $24 \%$ of women ( $n=10 / 41$ ), which was not different between groups and 4 women (10\%) exhibited both, reduced handgrip strength and sarcopenia. Findings from the abPG-SGA showed that nutrition impact symptoms were more frequently endorsed among those classified as non-sarcopenic vs. sarcopenic ( $p=0.08$, respectively.) Fatigue and pain were reported by $100 \%$ ( $n=41$ ) and $85 \%$ (36) of all participants, respectively. No differences in fruit, vegetable, fiber intake, fatigue or pain were detected between groups. In general, women with sarcopenia had higher total scores on both the FACT-Breast and the FACT-Endocrine Symptoms. When the subscales of these instruments were analyzed, non-sarcopenic women reported 
lower endocrine symptoms scores compared to sarcopenic women, respectively $(p=0.01)$. (Data not shown) Serum biomarkers of inflammation showed trends toward higher CRP in nonsarcopenic vs. sarcopenia women $(p=0.09)$.

Table 3 depicts the differences in body composition, lifestyle factors, QOL, symptoms and serum biomarkers of inflammation stratified by obesity status using BMI. Overall, $34 \%$ ( $n=14 / 41$ ) were classified as obese and myosteatosis occurred nearly equally among the women with normal/overweight vs. with obesity. Women with obesity had significantly greater body weight $(p=<0.0001)$, whole body SM $(p<0.01)$, reduced SMHU $(p=0.02)$ and abdominal obesity (all p values $<0.0001$ ), and reported less time engaged in leisure time exercise activity $(p=0.07)$ compared to women without obesity. Women with obesity also had significantly lower QOL scores for breast $(p=0.07)$ and endocrine symptoms $(p=0.02)$, as well as daily pain $(p=0.09)$ when compared to women without obesity. When the subscales of the QOL instruments were analyzed, women with obesity tended to reported lower physical and emotional well-being vs. women with normal/overweight. (Data not shown) Significantly higher levels of CRP ( $p=0.06)$ and TNF- $\alpha(p=0.03)$ were observed for women with obesity vs. without obesity. No differences in fruit, vegetable, fiber intake or handgrip strength was detected between obesity groups.

\section{Discussion:}

This exploratory study found that sarcopenia was prevalent in one of three women with ER+ metastatic BC. Our findings are consistent with Prado et al (15), but lower than the $58 \%$ prevalence reported by Shachar et al. (16) Our study is different, however, in that we only recruited women with $\mathrm{ER}+$ metastatic $\mathrm{BC}$. Additionally, our participants were at various phases of their disease course and we explored potential associations with patient-reported outcomes 
in 'real' time. We believe our findings may underestimate the occurrence of sarcopenia in this patient population since women were required to be clinically stable in order to participate in the parent investigation. Compared to a broader group of women with metastatic $\mathrm{BC}$ receiving more varied treatment regimens or undergoing more frequent $\mathrm{CT}$ imaging due to disease activity, our participants may be inherently "healthier." Interestingly when we compare our QOL findings to a larger normative group of women with metastatic $B C(n=98)(30)$, our women with sarcopenia have very similar mean scores for physical (22 vs. 22) and functional well-being (19 vs. 19), as well as total scores for the FACT-B (115 vs. 109) and FACT-ES (172 vs. 174), respectively. Although these findings are in contrast to our proposed hypotheses, women in our trial do reflect the needs and characteristics of those whom we would recruit for a lifestyle intervention targeting improvements in symptom management. Thus, these results make an important contribution to the limited literature on this growing population of survivors. Previous studies have shown that sarcopenia adversely impacts chemotherapy toxicity and/or overall survival in pancreatic $(36)$, renal $(37,38)$, respiratory, and gastrointestinal cancers. (39) Two studies have specifically examined oncology-related outcomes in women with metastatic BC. Prado et al investigated the clinical implications of SM in 55 women with ER+ and ER- metastatic BC starting capecitabine chemotherapy treatment. Women who were sarcopenic $\left(\mathrm{SM}<38.5 \mathrm{~cm}^{2} / \mathrm{m}^{2}\right)$ at treatment initiation $(\mathrm{n}=14 / 55)$ exhibited significant chemotherapy toxicities and shorter time to tumor progression compared to those with higher levels of SM. (15) Further, Shachar et al investigated the associations between sarcopenia and conventional oncology outcomes in 40 women with both ER+ and ER- metastatic BC receiving first line taxane-based chemotherapy. (16) These authors reported significantly higher toxicity- 
related hospitalizations and more severe adverse events (i.e., grade $3-4$ toxicities, hospitalizations, dose reductions, or dose delay) in women with sarcopenia ( $n=23)$ vs. those without $(n=17)$. Additionally, these investigators reported that myosteotosis was also associated with grade 3-4 toxicity, the need for hospitalization and time to treatment failure (for progression or toxicity) (all p values $<0.04$ ). Because chemotherapy dosing relies on body surface area using current height and weight, these calculations presume a constant proportion of adipose to lean tissue. In the setting of sarcopenia, these body composition assumptions are violated. As suggested by Prado et al, decreased levels of SM reflect a lower volume of chemotherapy drug distribution, resulting in greater toxicity reports, dose delays or treatment cessation. (39) While this concept is beyond the reach of the current study, it is clear that SM is an important prognostic tissue biomarker for women with metastatic BC. As discussed by the Cruz-Jentoft et al on behalf of the International Sarcopenia Initiative (40), identifying effective ways to improve SM is difficult; however, such interventions could have profound implications on treatment tolerance and survival for this clinical population.

While the primary focus of this study was SM, our stratification by obesity highlighted some intriguing findings. In particular, the concept that women classified with obesity experience a tendency toward greater symptomology and lower multidimensional QOL when compared to women with normal weight or overweight. BMI is considered a surrogate marker of body composition, specifically adiposity $(41,42)$ and many studies have demonstrated the high variability of SM in different oncology populations, independent of BMI categories. (7, 39, 43-45) Considering that the majority of our women with sarcopenia fell within the normal BMI range $(18.5-24.9 \mathrm{~kg} / \mathrm{m} 2)$ and the low prevalence of sarcopenic obesity $(n=1)$, leaves us to 
speculate on other potential mechanisms besides sarcopenia. Because women with obesity were found to have significantly lower SM quality (using SMHU; $p=0.02$ ), we analyzed fatigue, pain and multidimensional QOL stratified by myosteatosis. No relationships were revealed. Previous studies have demonstrated that persistent elevations in circulating pro-inflammatory cytokines [e.g., IL-6, TNF- $\alpha$ ] are observed in early stage BC survivors for years after treatment and are associated with adverse symptomology and reduced QOL. $(46,47)$ Based on the significantly higher levels of CRP, TNF- $\alpha$, VAT, SAT and TAT in our women classified with obesity vs. those without, the potential role of inflammation merits further exploration.

An important, yet subtle finding of this study is the fact that sarcopenia was present in women considered at 'low' nutrition risk, when using the abPG-SGA as a nutrition screening tool. In a previous study by Gabrielson et al (21), these authors reported that scores $\geq 6$ on the abPG-SGA were highly correlated with oncology patients classified as malnourished when the full versions of the PG-SGA ( $97 \%$ sensitivity, $86 \%$ specificity, AUC $=0.967)$ and the Malnutrition Screening Tool ( $81 \%$ sensitivity, $72 \%$ specificity, AUC $=0.823)$ were administered. Several other investigators have also demonstrated that inabilities of other common nutrition screening tools, including the Malnutrition Universal Screening Tool (MUST), Malnutrition Screening Tool (MST), and the Nutritional Risk Index (NRI), to detect compromised body composition in a heterogeneous group of oncology populations. (48-50) In direct contrast to our study, these investigations included patients with cancer cachexia; a population deemed ineligible for our larger study. Despite these differences and the fact that our study specifically applied the abPGSGA, a nutrition screen tool designed for the out-patient oncology population, this growing body of work highlights the limitations of these widely implemented nutrition screening tools. A 
particular challenge for oncology practitioners is the inability to detect SM depletion among those with overweight or obesity. Unlike cancer cachexia, where there are more overt signs of wasting (i.e., subjective complaints of unintentional weight loss, global adipose tissue loss, muscle wasting, etc.), sarcopenia is an occult condition not detectable upon physical examination or supported with questions taken during a routine history. In patients with overweight or obesity, mild changes in weight go unnoticed or are so subtle, they are missed even by experienced practitioners. In addition, the scale may show no net change as SM deteriorates and adipose tissue increases. Thus, it is becoming increasingly paramount to try to identify methods to incorporate imaging into our modern nutrition assessment techniques, as reiterated by these study results.

This study is not without limitations and a few points merit highlighting. First, we used CT images that were closest to the time of our initial study assessment. Previous investigators have used a 30-45 day window to correlate physical measures (e.g., body weight, BMI) with CT imaging to examine outcomes. $(7,39)$ However, we reasoned that a larger window for assessment was allowable because: 1) anthropometric measures and symptom assessments were obtained in 'real time' vs. retrospectively calculated from the EMR, and 2) our eligibility criteria eliminated women experiencing unintentional weight loss, cachectic in appearance and with new clinical or radiologic evidence of disease progression. While this provided a unique opportunity to explore body composition in this patient population, the larger window of time between questionnaire completion and CT imaging (range 0-159 days) may have unknowingly impeded our abilities to link important patient-reported outcomes with SM. Second, we only had 41 women included in these analyses and we may be statistically underpowered to detect 
differences in outcomes between groups. We also examined CT-detected sarcopenia, as it was not practical to explore outcomes for the 4 women who met the diagnostic criterion for sarcopenia, which includes reduced SM and compromised muscle strength. (35) Studies that are adequately powered are warranted to better explore the potential relationships between patient-reported outcomes and SM. Third, a fruit and vegetable screener was completed to evaluate dietary intake due to its ease and low burden relative to other study activities; however, more detailed dietary assessment methodologies (e.g., food records, food frequency questionniares) are needed to support a comprehensive nutrition assessment. Fourth, handgrip strength was used to obtain a simple, quantifiable measure of physical functioning. Likely more in-depth assessments of overall physical conditioning and functioning are needed to establish associations with lean mass and general fitness, similar to Jones et al (51) or Yee at al (52) in women with metastatic BC. Finally, this is a cross-sectional study and the data can only be used to for hypothesis generation; no cause and effect relationships can be established

\section{Conclusions:}

The rising incidence of metastatic $B C$ at initial diagnosis combined with expanding treatment options have resulted in a steadily growing population of women living with this disease. $(4,5,53)$ Our study continues to support the wide-spread prevalence of CT-detected sarcopenia and myosteatosis, now documenting their occurrence in an exclusive population of ER+ women. Our findings also reiterate the importance and potentially intertwined relationship of obesity, inflammation and QOL. Future interventions supported by multidisciplinary team efforts are needed to improve the body composition of women with $E R+$ metastatic $B C$ in the hopes of potentially improving a broad array of relevant outcomes. Additionally, studies 
gathering data at more than one time point would provide important opportunities to observe the trajectories of change during the time course of survivorship.

Acknowledgements: We acknowledge the efforts of Dr. Ellen Gaynor for her assistance with this study. 


\section{References}

1 Irvin W, Jr., Muss HB, Mayer DK: Symptom management in metastatic breast cancer. The oncologist 16, 1203-14, 2011. doi: 10.1634/theoncologist.2011-0159

$2 \quad$ National Cancer Institute, Surveillance Epidemiology and End Results (SEER) Program, Cancer Stat Facts: Female Breast Cancer, February 5, 2018.

3 American Cancer Society, American Cancer Society Cancer Facts and Figures 2018, January 12, 2018.

$4 \quad$ Finn RS, Martin M, Rugo HS, Jones S, Im SA, et al.: Palbociclib and Letrozole in Advanced Breast Cancer. N Engl J Med 375, 1925-1936, 2016. doi: 10.1056/NEJMoa1607303

5 Swain SM, Kim SB, Cortes J, Ro J, Semiglazov V, et al.: Pertuzumab, trastuzumab, and docetaxel for HER2-positive metastatic breast cancer (CLEOPATRA study): overall survival results from a randomised, double-blind, placebo-controlled, phase 3 study. The Lancet Oncology 14, 461-71, 2013. doi: 10.1016/S1470-2045(13)70130-X

6 Mariotto AB, Etzioni R, Hurlbert M, Penberthy L, Mayer M: Estimation of the Number of Women Living with Metastatic Breast Cancer in the United States. Cancer Epidemiol Biomarkers Prev 26, 809815, 2017. doi: 10.1158/1055-9965.EPI-16-0889

7 Martin L, Birdsell L, Macdonald N, Reiman T, Clandinin MT, et al.: Cancer cachexia in the age of obesity: skeletal muscle depletion is a powerful prognostic factor, independent of body mass index. Journal of clinical oncology : official journal of the American Society of Clinical Oncology 31, 1539-47, 2013. doi: 10.1200/JCO.2012.45.2722

8 Sheean PM, Hoskins K, Stolley M: Body composition changes in females treated for breast cancer: a review of the evidence. Breast Cancer Res Treat 135, 663-80, 2012. doi: 10.1007/s10549-0122200-8

9 Cheney CL, Mahloch J, Freeny P: Computerized tomography assessment of women with weight changes associated with adjuvant treatment for breast cancer. Am J Clin Nutr 66, 141-6, 1997

10 Demark-Wahnefried W, Hars V, Conaway MR, Havlin K, Rimer BK, et al.: Reduced rates of metabolism and decreased physical activity in breast cancer patients receiving adjuvant chemotherapy. Am J Clin Nutr 65, 1495-501, 1997

11 Demark-Wahnefried W, Peterson BL, Winer EP, Marks L, Aziz N, et al.: Changes in weight, body composition, and factors influencing energy balance among premenopausal breast cancer patients receiving adjuvant chemotherapy. J Clin Oncol 19, 2381-9, 2001

12 Gordon AM, Hurwitz S, Shapiro CL, LeBoff MS: Premature ovarian failure and body composition changes with adjuvant chemotherapy for breast cancer. Menopause 18, 1244-8, 2011. doi: 10.1097/gme.0b013e31821b849b

13 Kutynec CL, McCargar L, Barr SI, Hislop TG: Energy balance in women with breast cancer during adjuvant treatment. J Am Diet Assoc 99, 1222-7, 1999

14 Nissen MJ, Shapiro A, Swenson KK: Changes in weight and body composition in women receiving chemotherapy for breast cancer. Clin Breast Cancer 11, 52-60, 2011. doi: N8922TX10676KJOM [pii]

\subsection{6/CBC.2011.n.009}

15 Prado CM, Baracos VE, McCargar L, Reiman T, Mourtzakis M, et al.: Sarcopenia as a determinant of chemotherapy toxicity and time to tumor progression in metastatic breast cancer patients receiving capecitabine treatment. Clin Cancer Res 15, 2920-6, 2009

16 Shachar SS, Deal AM, Weinberg M, Nyrop KA, Williams GR, et al.: Skeletal Muscle Measures as Predictors of Toxicity, Hospitalization, and Survival in Patients with Metastatic Breast Cancer Receiving Taxane-Based Chemotherapy. Clin Cancer Res 23, 658-665, 2017. doi: 10.1158/1078-0432.CCR-16-0940 
17 Sheean P, Kabir C, Rao R, Hoskins K, Stolley M: Exploring Diet, Physical Activity, and Quality of Life in Females with Metastatic Breast Cancer: A Pilot Study to Support Future Intervention. J Acad Nutr Diet 115, 1690-8, 2015. doi: 10.1016/j.jand.2015.03.017

18 Oken MM, Creech RH, Tormey DC, Horton J, Davis TE, et al.: Toxicity and response criteria of the Eastern Cooperative Oncology Group. American journal of clinical oncology 5, 649-55, 1982

19 Prevention CfDCa. Classifications of Obesity, Overweight and Underweight Adults.

20 Detsky AS, McLaughlin JR, Baker JP, Johnston N, Whittaker S, et al.: What is subjective global assessment of nutritional status? JPEN J Parenter Enteral Nutr 11, 8-13, 1987

21 Gabrielson DK, Scaffidi D, Leung E, Stoyanoff L, Robinson J, et al.: Use of an abridged scored Patient-Generated Subjective Global Assessment (abPG-SGA) as a nutritional screening tool for cancer patients in an outpatient setting. Nutr Cancer 65, 234-9, 2013. doi: 10.1080/01635581.2013.755554 22 Block G, Gillespie C, Rosenbaum EH, Jenson C: A rapid food screener to assess fat and fruit and vegetable intake. Am J Prev Med 18, 284-8, 2000

23 Block Fruit/Vegetable/Fiber Screener Nutrition Quest.

24 Mourtzakis M, Prado CM, Lieffers JR, Reiman T, McCargar L, et al.: A practical and precise approach to quantification of body composition in cancer patients using computed tomography images acquired during routine care. Appl Physiol Nutr Metab 33, 997-1006, 2008. doi: h08-075 [pii]

10.1139/H08-075

25 Kelley DE, Slasky BS, Janosky J: Skeletal muscle density: effects of obesity and non-insulindependent diabetes mellitus. Am J Clin Nutr 54, 509-15, 1991

26 Goodpaster BH, Thaete FL, Simoneau JA, Kelley DE: Subcutaneous abdominal fat and thigh muscle composition predict insulin sensitivity independently of visceral fat. Diabetes 46, 1579-85, 1997

27 Goodpaster BH, Thaete FL, Kelley DE: Composition of skeletal muscle evaluated with computed tomography. Ann N Y Acad Sci 904, 18-24, 2000

28 Schlecht I, Fischer B, Behrens G, Leitzmann MF: Relations of Visceral and Abdominal Subcutaneous Adipose Tissue, Body Mass Index, and Waist Circumference to Serum Concentrations of Parameters of Chronic Inflammation. Obes Facts 9, 144-57, 2016. doi: 10.1159/000443691

29 Brady MJ, Cella DF, Mo F, Bonomi AE, Tulsky DS, et al.: Reliability and validity of the Functional Assessment of Cancer Therapy-Breast quality-of-life instrument. Journal of clinical oncology : official journal of the American Society of Clinical Oncology 15, 974-86, 1997

30 Fallowfield $\amalg$, Leaity SK, Howell A, Benson S, Cella D: Assessment of quality of life in women undergoing hormonal therapy for breast cancer: validation of an endocrine symptom subscale for the FACT-B. Breast Cancer Research and Treatment 55, 189-99, 1999

31 Cleeland CS, Ryan KM: Pain assessment: global use of the Brief Pain Inventory. Annals of the Academy of Medicine, Singapore 23, 129-38, 1994

32 Piper BF, Dibble SL, Dodd MJ, Weiss MC, Slaughter RE, et al.: The revised Piper Fatigue Scale: psychometric evaluation in women with breast cancer. Oncol Nurs Forum 25, 677-84, 1998

33 Godin G, Shephard RJ: A simple method to assess exercise behavior in the community. Canadian journal of applied sport sciences Journal canadien des sciences appliquees au sport 10, 141-6, 1985

34 Toolbox N. Performing hand grip strength measures NIH, 2012.

35 Cruz-Jentoft AJ, Baeyens JP, Bauer JM, Boirie Y, Cederholm T, et al.: Sarcopenia: European consensus on definition and diagnosis: Report of the European Working Group on Sarcopenia in Older People. Age and ageing 39, 412-23, 2010. doi: 10.1093/ageing/afq034

36 Tan BH, Birdsell LA, Martin L, Baracos VE, Fearon KC: Sarcopenia in an overweight or obese patient is an adverse prognostic factor in pancreatic cancer. Clin Cancer Res 15, 6973-9, 2009

37 Fukushima H, Nakanishi Y, Kataoka M, Tobisu K, Koga F: Prognostic Significance of Sarcopenia in Patients with Metastatic Renal Cell Carcinoma. J Urol 195, 26-32, 2016. doi: 10.1016/j.juro.2015.08.071 
38 Antoun S, Baracos VE, Birdsell L, Escudier B, Sawyer MB: Low body mass index and sarcopenia associated with dose-limiting toxicity of sorafenib in patients with renal cell carcinoma. Annals of oncology : official journal of the European Society for Medical Oncology / ESMO 21, 1594-8, 2010. doi: 10.1093/annonc/mdp605

39 Prado CM, Lieffers JR, McCargar L, Reiman T, Sawyer MB, et al.: Prevalence and clinical implications of sarcopenic obesity in patients with solid tumours of the respiratory and gastrointestinal tracts: a population-based study. Lancet Oncol 9, 629-35, 2008. doi: S1470-2045(08)70153-0 [pii]

10.1016/S1470-2045(08)70153-0

40 Cruz-Jentoft AJ, Landi F, Schneider SM, Zuniga C, Arai H, et al.: Prevalence of and interventions for sarcopenia in ageing adults: a systematic review. Report of the International Sarcopenia Initiative (EWGSOP and IWGS). Age Ageing 43, 748-59, 2014. doi: 10.1093/ageing/afu115

41 Wortsman J, Matsuoka LY, Chen TC, Lu Z, Holick MF: Decreased bioavailability of vitamin D in obesity. Am J Clin Nutr 72, 690-3, 2000

42 Cipriani C, Pepe J, Piemonte S, Colangelo L, Cilli M, et al.: Vitamin d and its relationship with obesity and muscle. Int J Endocrinol 2014, 841248, 2014. doi: 10.1155/2014/841248

43 Baracos VE, Reiman T, Mourtzakis M, Gioulbasanis I, Antoun S: Body composition in patients with non-small cell lung cancer: a contemporary view of cancer cachexia with the use of computed tomography image analysis. Am J Clin Nutr 91, 1133S-1137S, 2010. doi: 10.3945/ajcn.2010.28608C

ajcn.2010.28608C [pii]

$44 \quad$ Lieffers JR, Bathe OF, Fassbender K, Winget M, Baracos VE: Sarcopenia is associated with postoperative infection and delayed recovery from colorectal cancer resection surgery. Br J Cancer 107, 931-6, 2012. doi: 10.1038/bjc. 2012.350

45 Cushen SJ, Power DG, Teo MY, Maceneaney P, Maher MM, et al.: Body Composition by Computed Tomography as a Predictor of Toxicity in Patients With Renal Cell Carcinoma Treated With Sunitinib. Am J Clin Oncol, 2014. doi: 10.1097/COC.0000000000000061

46 Collado-Hidalgo A, Bower JE, Ganz PA, Cole SW, Irwin MR: Inflammatory biomarkers for persistent fatigue in breast cancer survivors. Clin Cancer Res 12, 2759-66, 2006. doi: 10.1158/10780432.CCR-05-2398

47 Jehn CF, Flath B, Strux A, Krebs M, Possinger K, et al.: Influence of age, performance status, cancer activity, and IL- 6 on anxiety and depression in patients with metastatic breast cancer. Breast Cancer Res Treat 136, 789-94, 2012. doi: 10.1007/s10549-012-2311-2

48 Ni Bhuachalla EB, Daly LE, Power DG, Cushen SJ, MacEneaney P, et al.: Computed tomography diagnosed cachexia and sarcopenia in 725 oncology patients: is nutritional screening capturing hidden malnutrition? J Cachexia Sarcopenia Muscle 9, 295-305, 2018. doi: 10.1002/jcsm.12258

49 Saroul N, Pastourel R, Mulliez A, Farigon N, Dupuch V, et al.: Which Assessment Method of Malnutrition in Head and Neck Cancer? Otolaryngol Head Neck Surg 158, 1065-1071, 2018. doi:

10.1177/0194599818755995

50 Thoresen L, Frykholm G, Lydersen S, Ulveland H, Baracos V, et al.: Nutritional status, cachexia and survival in patients with advanced colorectal carcinoma. Different assessment criteria for nutritional status provide unequal results. Clin Nutr 32, 65-72, 2013. doi: 10.1016/j.clnu.2012.05.009

51 Jones LW, Courneya KS, Mackey JR, Muss HB, Pituskin EN, et al.: Cardiopulmonary function and age-related decline across the breast cancer survivorship continuum. Journal of clinical oncology :

official journal of the American Society of Clinical Oncology 30, 2530-7, 2012. doi: 10.1200/JCO.2011.39.9014

52 Yee J, Davis GM, Beith JM, Wilcken N, Currow D, et al.: Physical activity and fitness in women with metastatic breast cancer. J Cancer Surviv 8, 647-56, 2014. doi: 10.1007/s11764-014-0378-y 
53 Johnson $\mathrm{RH}$, Chien FL, Bleyer A: Incidence of breast cancer with distant involvement among women in the United States, 1976 to 2009. JAMA : the journal of the American Medical Association 309, 800-5, 2013. doi: 10.1001/jama.2013.776 